\title{
Quel prix les habitants d'une région périurbaine payent-ils pour voir des paysages " verts " depuis chez eux ?
}

\author{
Jean Cavailhès $^{1}$ \\ Thierry Brossard ${ }^{2}$ \\ Jean-Christophe Foltête ${ }^{2}$ \\ Mohamed Hilal ${ }^{3}$ \\ Daniel Joly ${ }^{2}$ \\ François-Pierre Tourneux ${ }^{2}$ \\ Céline Tritz ${ }^{2}$ \\ Pierre Wavresky ${ }^{3}$ \\ ${ }^{1}$ Inra \\ UMR 1041 Cesaer \\ 26, boulevard Docteur-Petitjean \\ BP 87999 \\ 21079 Dijon cedex \\ France \\ <Jean.Cavailhes@enesad.inra.fr> \\ ${ }^{2}$ CNRS \\ ThéMA \\ 32, rue Megevand \\ 25030 Besançon \\ France \\ ${ }^{3}$ Inra \\ Cesaer \\ 21065 Dijon \\ France
}

\begin{abstract}
Résumé
L'objectif de cet article est d'estimer le prix d'attributs paysagers dans la région de Dijon. À partir d'images satellites et d'un modèle numérique de terrain, une méthode de géographie quantitative développée à partir de systèmes d'information géographique (SIG) permet de "mesurer " les paysages vus depuis le sol à travers une batterie d'indices. Sur la base de 2667 transactions immobilières, un modèle économétrique utilisant la méthode des prix hédonistes permet d'estimer les prix des attributs paysagers ainsi quantifiés. Les résultats montrent, tout d'abord, que dans cette région les ménages sont "myopes " : le prix de paysages vus au-delà d'une ou deux centaines de mètres n'est pas significativement différent de zéro. Dans la ceinture périurbaine et à proximité des habitations, les couverts arborés et agricoles ont des prix positifs, et les routes des prix négatifs lorsqu'ils sont vus depuis les maisons, mais ces prix sont plus faibles lorsque ces attributs ne sont pas vus. L'agencement des objets dans des compositions paysagères aux formes complexes (fragmentation, mosaïque, etc.) a également un prix positif. Il ressort de ces résultats que la fonction productive de l'agriculture et de la forêt est en conflit avec leur fonction paysagère, et que les politiques publiques visant à l'entretien des paysages doivent être différenciées selon la localisation par rapport aux villages et à l'habitat.
\end{abstract}

Mots clés : analyse économique ; entretien du paysage ; milieu périurbain ; paysage agricole ; système d'information géographique.

Thèmes : économie et développement rural ; ressources naturelles et environnement ; territoire, foncier, politique agricole et alimentaire.

\section{Abstract \\ How much do peri-urban inhabitants pay to see "green" landscapes in their vicinity?}

We estimate the price of landscape attributes in the urban fringe of Dijon (France). Landscapes as seen from the ground are analyzed from satellite images and from a digital elevation model developed from a geographic method using geographical information systems. An econometric model uses the hedonic pricing method, derived from the sales of 2,667 houses, to assess the landscape attributes identified by the geographical model. The results show that households are 'short-sighted': in this region, landscapes and visible features more than 100-200 $\mathrm{m}$ away all have insignificant hedonic prices. In the peri-urban belt, forests and farmland in the immediate vicinity of houses have positive prices and roads a negative price when these features can be seen, while their prices are lower when they cannot be seen. The arrangement of features in complex or fragmented landscapes commands a positive hedonic price. The results show that the productive function of agriculture and forestry is in contradiction with its scenic function, and that public policies directed at landscape maintenance should take the precise location into account with reference to the urban system.

Key words: agricultural landscape; economic analysis; geographical information systems; landscape maintenance; peri-urban environment.

Subjects: economy and rural development; natural resources and environment; territory, land use, agricultural and food production policy. 
$\mathrm{T}$ ravailler en ville tout en résidant à la campagne fait partie des modes de vie fréquents d'aujourd'hui. Aussi, la protection des paysages, en particulier ceux qui forment le cadre résidentiel des habitants proches des villes, fait l'objet d'une attention croissante. Cette préoccupation n'est pas nouvelle, mais elle fait maintenant l'objet d'un intérêt accru de la part des aménageurs, car l'extension périurbaine, qui caractérise les métropoles des pays développés depuis plusieurs décennies, ne cesse de s'accrô̂tre. C'est pourquoi les travaux économiques sur cette question se développent.

Nous estimons ici la valeur de paysages "verts " en utilisant la méthode des prix hédonistes, qui repose sur l'hypothèse que cette valeur se capitalise dans le prix de l'immobilier. La recherche porte sur la ceinture périurbaine de Dijon, large d'une quarantaine de kilomètres. Elle est constituée de quelques centaines de villages et petites villes disséminés dans une plaine agricole et sur des coteaux vallonnés où forêts, champs et prairies s'entremêlent. Il s'agit de paysages ruraux que l'on peut qualifier d'ordinaires, ce qui rend leur prix plus difficile à estimer que celui de sites remarquables.

La difficulté principale est de "mesurer " ces paysages par des variables à introduire dans une équation économétrique. À cette fin, nous avons eu recours à des méthodes de géographie quantitative et à des systèmes d'information géographique (SIG) où sont stockés des images satellites et des modèles numériques de terrain précis. Des protocoles spécifiques permettent d'analyser le paysage vu du sol, en tenant compte du relief et des objets qui masquent la vue. Cette recherche a bénéficié du financement de la région de Bourgogne, du Conseil général de la Côte-d'Or et de la Communauté d'agglomération de Dijon.

Nous présentons les modèles géographiques et économiques, les sources et les données dans la section 2 puis les résultats dans la section 3. La section 4 tire les conclusions.

\section{Modèles géographique et économique, données}

\section{Modèle géographique d'analyse des paysages}

Espace offert à la vue, le paysage se définit ici en termes quantitatifs (surface d'es- pace vu) et qualitatifs (nature des objets vus). Pour analyser cette réalité (Cavailhès et Joly, 2006), deux types d'information sont couplés : l'occupation du sol qui positionne et identifie les classes d'objets disposés à la surface terrestre et un modèle numérique de terrain (MNT) qui modélise la topographie. Le paysage est " reconstruit " par modélisation trigonométrique : chaque pixel de l'aire d'étude devient le point d'observation d'une scène paysagère de synthèse. Pour réduire les temps de calcul, le tour d'horizon est échantillonné selon 120 rayons simulant l'axe du regard. Le long de chaque axe, les pixels vus (en fonction de la configuration du relief et des objets présents au sol) sont dénombrés (figure 1). Pour tenir compte de l'effet de masque lié à ces objets, une hauteur standard est affectée à chaque type d'occupation du sol : sept mètres pour les maisons, 15 pour les feuillus, zéro pour l'eau ou les routes, etc.

La modélisation numérique tient compte de la profondeur de champ, car, avec l'éloignement, le regard ne parvient pas à discriminer, avec une précision constante, les composants du paysage. C'est pourquoi notre modèle de visibilité décom- pose le paysage visible en quatre plans successifs qui déterminent autant d'auréoles concentriques dont les limites sont : 0-280 m, 281-1 $200 \mathrm{~m}, 1201-6000 \mathrm{~m}$, $6-40 \mathrm{~km}$. Chacune d'elles est connectée à une base de données dont la résolution est différente, soit : 7, 30, 150, et $1000 \mathrm{~m}$. De la sorte, la précision thématique et géométrique de l'information requise pour analyser, plan par plan, les propriétés visuelles du paysage est modulée en fonction de l'éloignement :

- à 7 et $30 \mathrm{~m}$ de résolution, l'occupation du sol est tirée du traitement combiné d'images provenant de deux satellites Landstat 7 ETM et Indian Remote Sensing, 12 thèmes étant distingués : eau, résineux, feuillus, buissons, cultures, prés, vignes, routes, bâti, carrières, voies ferrées et zones d'activité ;

- à 150 et $1000 \mathrm{~m}$ de résolution, l'information provient de la base de données Corine Land Cover. Une simplification de la nomenclature initiale de cette base de données a été opérée : huit thèmes d'occupation du sol sont conservés à la résolution de $150 \mathrm{~m}$ (eau, résineux, feuillus, buissons, cultures, prés, vignes, bâti) et quatre seulement à $1000 \mathrm{~m}$ (eau, forêt, espace agricole, bâti).

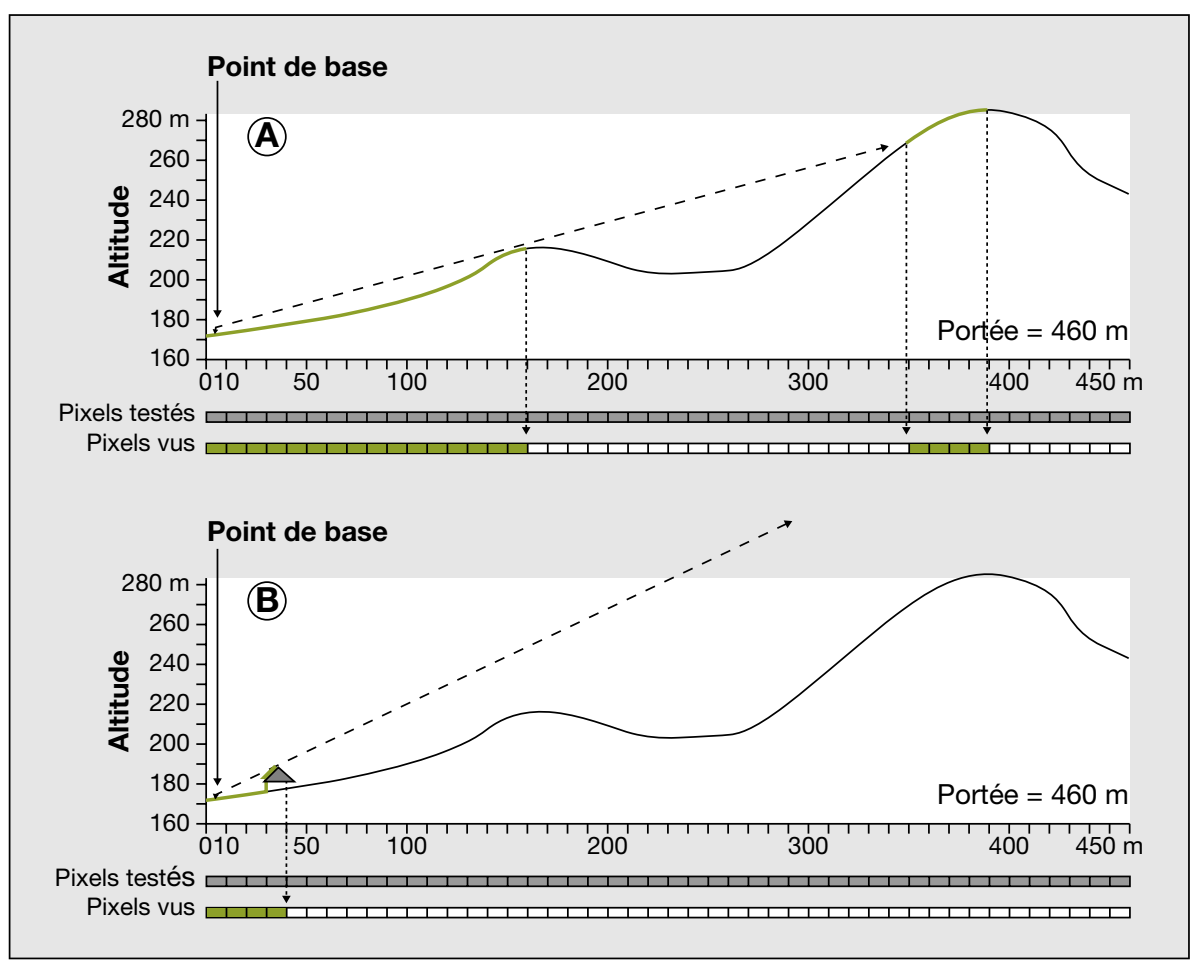

Figure 1. Le paysage vu du sol selon le relief $(A)$ et les objets hauts (B)

Figure 1. The viewshed according to the relief $(A)$ and the high objects (B). 
Les MNT sont issus de la "BD-ALTI " de l'Institut national géographique (IGN). Les reliefs de la région se caractérisant par des courbes (convexes, concaves) très lissées, nous avons appliqué une interpolation gravitaire au MNT initial à $50 \mathrm{~m}$ de résolution pour produire les couches à 7 et $30 \mathrm{~m}$. Ces dernières, ne décrivent évidemment pas les fines variations topographiques dont l'incidence sur les rapports d'intervisibilité est faible, voire négligeable au regard des formes d'occupation du sol (Joly et al., 2009). Les altitudes sont exprimées en unité centimétrique afin d'assurer une bonne description des éléments de pente faible ou de glacis, communs aux abords de la Saône. Aux échelles supérieures, une procédure d'agrégation a permis de dériver les couches à 150 et à $1000 \mathrm{~m}$ des MNT initiaux à 50 et $250 \mathrm{~m}$.

On obtient finalement des matrices où les lignes (2 667 au total) correspondent aux transactions, tandis que les colonnes définissent les attributs paysagers influant sur le prix des biens. C'est cet aspect économique de l'évaluation du paysage qui est abordé maintenant.

\section{Modèle économique de prix hédonistes}

Un logement est un bien complexe constitué d'attributs intrinsèques (taille, date de construction, confort, équipements, etc.) et d'une localisation aux caractéristiques particulières, décrite par d'autres attributs, parmi lesquels les attributs paysagers qui nous intéressent particulièrement. Le prix du logement est une combinaison de la valeur de l'ensemble de ces attributs. Tout se passe comme si le ménage achetait, dans le respect de sa contrainte budgétaire, un logement offrant la proportion optimale d'attributs, compte tenu de leurs prix respectifs. L'idée de départ est donc simple : le prix d'un bien est égal à la somme des prix de ses attributs multipliés par leurs quantités. Cette méthode soulève des problèmes théoriques et statistiques, évoqués ici en quelques mots. Premièrement, il est possible que l'acheteur choisisse simultanément le prix de la maison et la quantité de certains attributs (par exemple : le nombre de pièces). Dans ce cas, la relation causale est dans les deux sens, alors que la méthode économétrique nécessite d'estimer une variable (le prix) par des variables explicatives indépendantes. Nous allons indiquer comment nous résolvons ce problème. Deuxièmement, il peut exister des liaisons spatiales entre les résidus de l'équation économétrique, dues à des caractéristiques omises que partagent des observations voisines (politique foncière locale, biens publics locaux, etc.). Pour en tenir compte, nous estimons un modèle "à effets fixes " dans lequel une variable spécifique est affectée à chaque commune qui capte toutes ces caractéristiques omises. Nous vérifions, par un test statistique de nullité de l'indice de Moran, qu'il n'existe pas d'autres corrélations entre les résidus. Troisièmement, les variables explicatives peuvent être corrélées entre elles, ce qui se traduit par des estimateurs inconsistants ; des transformations de variables permettent de réduire l'importance de ce problème.

Au total, nous estimons une équation loglinéaire : le logarithme du prix d'une maison est expliqué par la somme de quantités d'attributs, parmi lesquels les caractéristiques intrinsèques de la maison ( $c f$. ciaprès les principales variables), la localisation dans une commune donnée de la région d'étude (c'est-à-dire un modèle à effets fixes) et la quantité des attributs paysagers, calculée comme il vient d'être dit. Seuls ces derniers résultats sont commentés ci-après. Les tests statistiques montrent que la surface habitable est endogène, ce qui conduit à projeter cette variable sur des variables "instrumentales " exogènes pour que toutes les variables explicatives soient exogènes (méthode instrumentale). Le test de nullité de l'indice de Moran montre qu'il n'y a pas d'autocorrélation spatiale des résidus. Les problèmes économétriques habituels de la méthode sont donc ainsi résolus.

Il faut, cependant, être prudent en lisant les résultats ; d'une part, il existe une marge d'erreur dans l'estimation d'un paramètre duquel on déduit un prix hédoniste (un "intervalle de confiance "); d'autre part, les résultats sont le fruit d'un modèle économétrique particulier, appliqué à des transactions d'une période donnée pour une région particulière. Dans une autre région et pour une autre période, des résultats différents pourraient sans doute être obtenus. D'ailleurs, pour la région que nous étudions, nous obtenons des prix hédonistes légèrement différents pour la ceinture périurbaine, comme ici, ou en incluant dans la région d'étude les communes de la banlieue dijonnaise ou encore selon l'équation économétrique retenue.

\section{Région d'étude et données}

\section{Région d'étude}

Région d'étude est délimitée par un temps d'accès à Dijon inférieur à 33 minutes ou une distance routière de moins de $42 \mathrm{~km}$ (les communes du pôle urbain de Dijon sont exclues) (figure 2). Elle présente quatre grands ensembles géographiques. Au nord de Dijon, s'étendent des plateaux calcaires avec de grandes exploitations céréalières extensives. Au sud de Dijon, trois bandes se succèdent : à l'ouest, l'Auxois est une région d'élevage avec un paysage de bocage organisé en vallées herbagères et croupes forestières ; ensuite, l'Arrière-Côte forme un plateau calcaire coupé de vallées sèches où l'agriculture est diversifiée (fruits, céréales, élevage) ; enfin, la plaine de la Saône où voisinent des zones forestières et des terres labourées avec des productions agricoles intensives (maraîchage et grandes cultures). Un talus abrupt sépare ces deux dernières régions, sur lequel s'étend une étroite bande viticole (la Côte-d'Or) qui donne sa réputation au vin de Bourgogne.

\section{Données économiques}

Elles proviennent des notaires, à partir des mutations immobilières. La base de données est constituée de 2667 ventes de maisons, intervenues entre 1995 et 2002. Il s'agit de ventes entre particuliers pour lesquelles les notaires relèvent le prix et certaines caractéristiques du bien et des acheteurs et vendeurs, en particulier : la surface habitable et celle de la parcelle, le nombre de pièces principales, celui de niveaux, la date de mutation, l'année ou l'époque de construction, le nombre de salles de bain, la présence de combles, sous-sol, piscine, parking, les modalités de la transaction, etc. Ce sont les " attributs intrinsèques " introduits dans l'équation.

\section{Variables paysagères}

Elles concernent les champs de vision et leur étendue, l'occupation des sols, la composition paysagère et la topographie. Nous mesurons les surfaces en are. En distinguant trois champs de vision dans les 280 premiers mètres (analysés avec la résolution de sept mètres : $c f .2 .1$ ), nous retenons au total six champs de vision : moins de $70 \mathrm{~m}$; entre 71 et $140 \mathrm{~m}$; entre 141 et $280 \mathrm{~m}$; entre 281 et $600 \mathrm{~m}$; entre $601 \mathrm{~m}$ et 1,2 km ; entre 1,2 et $6 \mathrm{~km}$ et plus de $6 \mathrm{~km}$.

Les cellules sont classées en 12 catégories, dont certaines sont regroupées : bâti, 


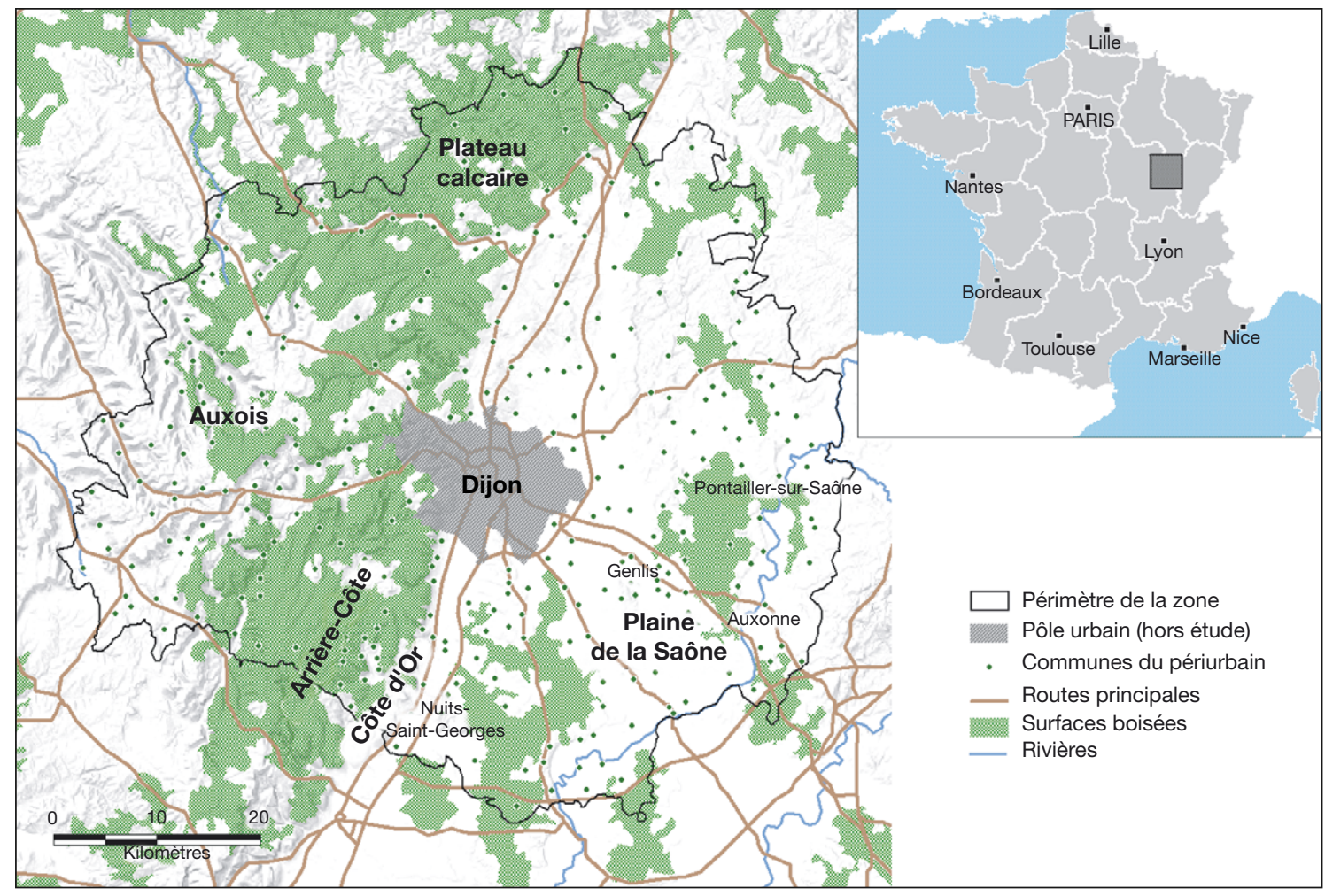

Figure 2. Région d'étude.

Figure 2. The study area.

buisson, carrière, eau, formation arborée (feuillus et résineux), agriculture (labour, prairie et vigne), route et voie ferrée, zone d'activité. Des variables de composition paysagère s'inspirent de l'écologie des paysages et sont calculées à partir de la base documentaire du logiciel FragStat (McGarigal et al., 2002) sur l'image d'occupation des sols en 12 classes.

\section{Résultats}

Nous ne présentons pas, ici, les résultats des variables non paysagères qui sont cohérents avec ceux obtenus par ailleurs (Cavailhès, 2005). Les paramètres de la régression économétrique et les prix hédonistes calculés à partir de ceux-ci sont présentés dans le tableau 1 pour les variables de localisation et paysagères.

\section{Couverts arborés et agricoles}

Un are arboré supplémentaire vu à moins de $70 \mathrm{~m}$ a un prix hédoniste de
1270 euros, soit 1,2\% du prix d'une maison. La forme des feuillus exerce également un effet significatif sur le prix, qui s'ajoute au précédent : une tache supplémentaire dans ce rayon de $70 \mathrm{~m}$ a un prix de 1190 euros et, à l'inverse, le prix de la longueur de leurs lisières est de -58 euros par mètre supplémentaire. La combinaison de ces deux variables donne une indication sur les formes qui sont valorisées : des taches nombreuses avec peu de lisières correspondent à de petits bosquets arrondis et non à des forêts en masse ni à des formations allongées.

Il est difficile de mettre ces résultats en regard de la littérature économique française, car celle-ci n'est pas unanime sur le prix hédoniste des formations arborées. La plupart des travaux anglo-saxons concluent que la proximité de forêts est appréciée (Thorsnes, 2002 ; Tyvainen et Miettinen, 2000 ; Des Rosiers et al., 2002), présentant une large bibliographie sur le sujet. Cependant, d'autres travaux présentent des résultats contraires, comme Smith et al. (2002) ; Irwin (2002) ; Garrod et Willis (1992).

Les cultures prés et vignes ont été agrégés dans une catégorie " agriculture ". Son paramètre n'est pas significatif pour les
70 premiers mètres, mais un are supplémentaire d'espaces agricoles vus entre 71 et $140 \mathrm{~m}$ des maisons a un prix hédoniste positif de 39 euros. La vue sur l'agriculture a donc un effet positif sur les valeurs immobilières, mais à une distance moins proche que celle des arbres, quoiqu'elle reste circonscrite à guère plus d'une centaine de mètres. Pour Smith et al. (2002), les ménages paient moins cher les logements situés près de terres agricoles, a fortiori lorsqu'ils sont adjacents. Sullivan et al. (2004) montrent l'intérêt de zones tampons arborées ou herbeuses entre les espaces agricoles et l'habitat, ce qui tend à confirmer qu'une trop grande proximité de l'agriculture déprécie les biens, résultat cohérent avec celui que nous obtenons. Notons également que les taches formées par l'agriculture en vue à une distance de 71 à $140 \mathrm{~m}$ ont un prix hédoniste de 270 euros par tache supplémentaire.

Les résultats montrent également que la vue proprement dite compte : le prix hédoniste de la simple présence de formations arborées à moins de 70 m d'une maison n'est que de 390 euros/are lorsqu'elles ne sont pas vues, soit trois fois moins que lorsqu'elles sont vues. Des 
Tableau 1. Résultats (variables paysagères)

Table 1. Results (landscape variables).

\begin{tabular}{|c|c|c|c|}
\hline Variable & Paramètre & $t$ de Student & $\begin{array}{l}\text { Prix hédoniste } \\
\text { (euros pour } 49 \mathrm{~m}^{2} \\
\text { ou par unité) }\end{array}$ \\
\hline$<200$ m d'une voie principale & $-0,07375^{* * *}$ & $-2,6$ & -7749 \\
\hline Zone UC ou UD du PLU (ex-POS) & $-0,03989 * * *$ & $-2,8$ & -4262 \\
\hline Zone mixte résidence-activité du POS & $-0,06415^{* *}$ & $-2,2$ & -6773 \\
\hline Distance à la mairie (mètres) & $-0,00004 * * *$ & $-2,4$ & $-4,4$ \\
\hline Orientation Sud & $0,000419 *$ & 1,9 & 46 \\
\hline Couverts arborés vus $<70 \mathrm{~m}\left(49 \mathrm{~m}^{2}\right)$ & $0,005694^{* * *}$ & 2,6 & 622 \\
\hline Couverts arborés vus $\left(49 \mathrm{~m}^{2}\right) *$ surface terrain $\left(\mathrm{m}^{2}\right)$ & $-0,00017^{*}$ & $-1,5$ & -19 \\
\hline Couverts arborés non vus $<70 \mathrm{~m}\left(49 \mathrm{~m}^{2}\right)$ & $0,001714^{* * *}$ & 3,5 & 187 \\
\hline Couverts arborés non vus $\left(49 \mathrm{~m}^{2}\right) *$ surface terrain $\left(\mathrm{m}^{2}\right)$ & $-0,00006 * * *$ & $-4,1$ & $-6,5$ \\
\hline Pourcentage de couverts arborés 71-140 m (49 $\left.\mathrm{m}^{2}\right)$ & 0,0005 & 0,0 & NS \\
\hline Couverts arborés $71-140$ m $\left(49 \mathrm{~m}^{2}\right)$ & $-0,0007$ & $-1,1$ & NS \\
\hline Pourcentage de buissons $<70 \mathrm{~m}$ & 0,026034 & 0,6 & NS \\
\hline Pourcentage de buissons $71-140 \mathrm{~m}$ & $0,244944^{* *}$ & 2,2 & 30253 \\
\hline Pourcentage de buissons 141-280 m & 0,085563 & 0,6 & NS \\
\hline Pourcentage d'agriculture vue $<70 \mathrm{~m}$ & $-0,01748$ & $-0,6$ & NS \\
\hline Pourcentage d'agriculture non vue $<70 \mathrm{~m}$ & 0,012073 & 0,2 & NS \\
\hline Agriculture vue $71-140 \mathrm{~m}\left(49 \mathrm{~m}^{2}\right)$ & $0,00017 * * *$ & 4,0 & 19 \\
\hline Agriculture vue $71-140 \mathrm{~m}\left(49 \mathrm{~m}^{2}\right) *$ surface terrain $\left(\mathrm{m}^{2}\right)$ & $-0,00639 * * *$ & $-5,7$ & -694 \\
\hline Agriculture vue $71-140 \mathrm{~m}\left(49 \mathrm{~m}^{2}\right) *$ zone $U$ des POS & $-0,00005^{*}$ & $-1,6$ & $-5,4$ \\
\hline Agriculture non vue $71-140 \mathrm{~m}\left(49 \mathrm{~m}^{2}\right)$ & $0,000036 * * *$ & 2,7 & 3,9 \\
\hline Agriculture non vue $71-140 \mathrm{~m}\left(49 \mathrm{~m}^{2}\right) *$ surface terrain $\left(\mathrm{m}^{2}\right)$ & $-0,00201 * * *$ & $-2,4$ & -219 \\
\hline Agriculture + arboré vu $\left(49 \mathrm{~m}^{2}\right)>140 \mathrm{~m}$ & 0,000024 & 0,3 & NS \\
\hline Bâti vu $<70 \mathrm{~m}\left(49 \mathrm{~m}^{2}\right)$ & 0,002041 & 1,6 & NS \\
\hline Pourcentage de bâti vu 71-280 m & $-0,0025$ & $-0,1$ & NS \\
\hline Pourcentage de bâti vu 281-1 200 m & 0,00488 & 0,1 & NS \\
\hline Routes + voies ferrées vues $\left(49 \mathrm{~m}^{2}\right)<280 \mathrm{~m}$ & $-0,00031^{* *}$ & $-2,2$ & -34 \\
\hline Routes + voies ferrées non vues $\left(49 \mathrm{~m}^{2}\right)<280 \mathrm{~m}$ & 0,000046 & 0,8 & NS \\
\hline Pourcentage de routes + voies ferrées vues $\left(49 \mathrm{~m}^{2}\right)$ 281-1 $200 \mathrm{~m}$ & $-0,25142$ & $-0,9$ & NS \\
\hline Eau vue $\left(49 \mathrm{~m}^{2}\right)$ & $-0,04184^{* *}$ & $-2,1$ & -4466 \\
\hline Lisières feuillues (mètres) & $-0,00053^{* * *}$ & $-3,6$ & -58 \\
\hline Nombre de taches de feuillus & $0,010867 * * *$ & 3,0 & 1191 \\
\hline Nombre de taches d'agriculture & $0,002487^{* * *}$ & 2,9 & 271 \\
\hline Indice de compacité & $0,231625^{*}$ & 1,7 & 28411 \\
\hline
\end{tabular}

Significativité : ** $1 \% ; * * 5 \% ; * 10 \%$; NS : non significatif.

forêts proches, quoique non vues, sont valorisées pour leurs fonctions récréatives (espace de promenade), de protection (contre le bruit), écologiques (qualité de l'air, faune et flore), etc., mais le prix est nettement inférieur à celui de formations arborées qui sont vues. Il en va de même pour l'agriculture non vue dans la couronne $71-140 \mathrm{~m}$, qui a un prix positif de huit euros pour un are supplémentaire, cinq fois moindre que celui de l'agriculture vue.

Des variables d'interaction entre le nombre de cellules arborées (vues à moins de
$70 \mathrm{~m}$ ) ou d'espaces agricoles (vus entre 71 et $140 \mathrm{~m}$ ) et la taille des lots résidentiels sont significativement négatives, ce qui indique que lorsqu'on voit beaucoup de forêt ou d'agriculture, le prix attaché à la surface de la parcelle est plus faible que lorsqu'on voit moins de ces aménités vertes. Il est probable qu'une relation de substituabilité entre ces deux types de biens existe : les couverts arborés ou agricoles ont donc une fonction land saving en limitant l'occupation résidentielle des sols.

Les buissons ont un prix non significatif, ce qui peut s'expliquer par l'hétérogénéité de cette catégorie (composée de haies, friches en cours de lignification, taillis, etc.). Enfin, au-delà des 70 premiers mètres pour les couverts arborés et de $140 \mathrm{~m}$ pour les espaces agricoles, toutes les variables testées ont des prix hédonistes non significatifs : il semble que, dans cette région, les ménages soient " myopes ".

Notons, enfin, qu'une interaction entre la vue d'espaces agricoles et la localisation dans une zone urbanisable du plan local d'urbanisme est significativement négative $(-20 \%)$, ce qui montre que les ména- 
ges accordent moins de valeur à cette vue dans une zone qui risque d'être urbanisée, entraînant ainsi la disparition de l'aménité agricole. La littérature internationale montre également le rôle du statut juridique des terres agricoles, qui définit des espaces pénétrables ou non (Cheshire et Sheppard, 1995) ou qui sont plus ou moins protégés d'une conversion résidentielle (Bockstael et Irwin, 2000 ; Irwin, 2002 ; Smith et al., 2002). Le prix hédoniste intègre les anticipations sur ces risques de conversion.

\section{Autres attributs paysagers et locaux}

La vue sur du bâti, occupation du sol la plus fréquente à proximité des habitations dans cette région d'habitat groupé, a un prix hédoniste non significatif quelle que soit la distance, peut-être à cause de deux effets opposés : la proximité d'autres habitations permet des relations sociales de voisinage, mais les ménages peuvent préférer voir des champs ou des bois plutôt que des constructions.

Les routes et voies ferrées vues dans les 70 premiers mètres autour des maisons ont un prix hédoniste négatif de 70 euros pour $100 \mathrm{~m}^{2}$ supplémentaires. En revanche, ces mêmes voies de communication n'ont pas d'effet significatif sur le prix des maisons, lorsqu'elles ne sont pas vues. Or, leur seule présence pourrait être une nuisance : bruit, pollution, source de danger, etc. Il semble qu'il n'en est rien, ce qui confirme les résultats obtenus pour l'agriculture et la forêt : la vue proprement dite compte plus que la seule présence. Cependant, la présence d'une voie rapide à moins de $200 \mathrm{~m}$ des habitations se traduit par une décote de 7750 euros.

D'autres variables de localisation intravillageoise influencent le prix des habitations : les maisons en zones classées comme mixtes (habitat et activités économiques) par les plans locaux d'urbanisme (PLU) valent 6770 euros de moins que les autres ; celles situées en périphérie de villages sont également moins chères (- 440 euros pour un éloignement de $100 \mathrm{~m}$ de la mairie, - 4260 euros pour les zones UC ou UD des PLU).

La vue de rivières semble être dévalorisée. Ce résultat, contraire à celui de la plupart des travaux anglo-saxons, est probablement dû au faible nombre de maisons depuis lesquelles on voit de l'eau. Les autres objets, comme les zones d'activité et les carrières, sont également trop peu représentés pour que les paramètres correspondants aient un sens.

Nous avons également introduit, dans l'équation économétrique, des variables de composition des paysages, parmi lesquels a été finalement retenu le nombre de taches de feuillus et d'agriculture, déjà évoqué, de même que la longueur des lisières de feuillus. Un indice de compacité, variant de 0 (formes très compactes) à 1 (formes allongées) a aussi été retenu, qui montre que pour $1 \%$ "d'élongation " en plus, le prix des maisons augmente de 284 euros. D'autres variables ont également été testées, qui montrent que la division des paysages, leur complexité ou leur fragmentation, les formes en mosaïque, etc. sont positivement valorisées.

\section{Conclusions:}

\section{les politiques agricoles et forestières}

Ces résultats concernent la seule région de Dijon. Il ne faut donc pas en tirer des conclusions de portée générale. Ils méritent néanmoins d'être portés au débat, car ils fournissent des hypothèses de travail nouvelles.

Les agriculteurs français se sont longtemps opposés au qualificatif de "jardiniers de la nature ", mais ils revendiquent aujourd'hui leur fonction d'entretien de la nature. L'une des raisons de ce changement est que les aides publiques pour l'entretien des paysages et de l'espace entrent dans la boîte verte des négociations de l'Organisation mondiale du commerce (OMC). Nos résultats apportent trois éclairages sur cette question.

Tout d'abord, les soutiens publics consacrés à l'agriculture et ceux à la forêt ne semblent pas correspondre au prix que les ménages accordent à chacun de ces paysages. Le prix hédoniste d'un are agricole vu (entre 141 et $280 \mathrm{~m}$ ) est 32 fois plus faible que celui d'un are de forêt vue (de 0 à $70 \mathrm{~m}$ ). Or, les aides publiques par are de forêt sont de 0,28 et celles en faveur de l'agriculture de 3,86 (Agreste, 2005), soit près de 14 fois plus. Certes, les aides à l'agriculture ne sont pas uniquement justifiées par leur rôle paysager ; le contraste est néanmoins frappant.
Ensuite, les aides publiques à l'agriculture et à la forêt sont faiblement liées à la localisation de ces activités par rapport à l'habitat, voire pas du tout. Or, c'est seulement les espaces verts très proches des habitations qui ont un prix hédoniste positif pour les habitants, alors que la grande majorité de ces activités sont situées au-delà de cet horizon. La fonction paysagère des usages agroforestiers des sols pour les habitants du périurbain ne peut donc être suffisante pour justifier le soutien public, au-delà d'une petite fraction de ces activités. Cependant, ces paysages agroforestiers sont également appréciés par des promeneurs, et ils ont une valeur patrimoniale.

Les résultats qui concernent les variables de composition paysagère vont dans le même sens. Depuis des décennies, le remembrement agricole a constitué de grandes parcelles aux formes simples pour faciliter le travail mécanique du sol ; les haies ont été rasées, les assolements végétaux simplifiés. Les forêts ont connu des évolutions semblables, quoique dans une moindre mesure : des forêts équiennes sur de vastes parcelles remplacent des plantations d'âges et d'espèces différentes, dans le même objectif d'accroître la productivité. Le résultat est la constitution de paysages uniformes et homogènes. Pourtant, les indices de composition paysagère que nous avons introduits montrent que ce sont des formes complexes, des mosaïques, de petites taches allongées, des paysages fragmentés qui sont valorisés par les ménages. Il y a, clairement, un contraste entre la fonction productive et la fonction paysagère de l'agriculture et de la forêt.

\section{Références}

Agreste, L'agriculture la forêt et les industries agro-alimentaires. Graph Agri 2005.

Bockstael NE, Irwin EG. Economics and the land use. Environment link. In: Tietinberg T, Folmer $\mathrm{H}$, eds. International yearbook of environmental and resource economics 2000-2001. Cheltenhan (UK) : Edward Edgar, 2000.

Cavailhès J. Le prix des attributs du logement. Econ Stat 2005; 381-382 : 91-123.

Cavailhès J, Joly D. Les paysages périurbains et leur prix. Besançon: Presses Universitaires de Franche-Comté, 2006.

Cheshire P, Sheppard S. On the price of land and the value of amenities. Economica 1995 ; 62 : 247-67.

Des Rosiers $F$, Thériault $M$, Kestens $Y$, Villeneuve $P$. Landscaping and house values: an empirical investigation. JReal Estate Res $2002 ; 23$ : 139-61. 
Garrod GD, Willis KG. Valuing goods' characteristics: an application of the hedonic price methos to environmental attributes. J Environ Manage $1992 ; 34$ : 59-76.

Irwin EG. The effects of open space on residential property values. Land Econ 2002; 78 (4) : 465-80.

Joly D, Brossard T, Cavailhès J, et al. Landscape modeling for a quantitative evaluation: an interdisciplinary approach. Annals of the Association of American Geographers 2009 (à paraître).
McGarigal K, Cushman SA, Neel MC, Ene E. Fragstats: spatial pattern analysis program for categorical maps. Computer software program produced by the authors at the University of Massachusetts, Amherst : University of Massachusetts, 2002. www.umass.edu/landeco/research/fragstats/fragstats.html.

Smith VK, Poulos C, Kim H. Treating open space as an urban amenity. Resour Energy Econ $2002 ; 24: 107-29$.
Sullivan WC, Anderson OM, Taylor Lovell S. Agricultural buffers at the urban-rural fringe: an examination of approval by farmers, residents and academics in the Midwestern United States. Landsc Urban Plan 2004 ; 69 : 299-313.

Thorsnes P. The value of a suburban forest preserve : Estimates from sales of vacant residential building lots. Land Econ 2002; 78 426-41.

Tyrvainen $L$, Miettinen A. Property prices and urban forest amenities. JEnviron Econ Manage 2000 ; 39 : 205-23. 